\title{
AVALIAÇÃO DA RELAÇÃO ENTRE SARCOPENIA E FUNCIONALIDADE EM IDOSAS QUILOMBOLAS DE BREJINHO DE NAZARÉ - TO
}

\author{
Evaluation of the relationship between sarcopenia and functionality in elderly \\ kilombolas of Brejinho de Nazaré - TO
}

Evaluación de la relación entre sarcopenia y funcionalidad en idosos quilombolas de Brejinho de Nazaré - TO

\section{Fellipe Camargo Ferreira Dias ${ }^{* 1}$, Luiz Sinesio Silva Neto}

${ }^{1}$ Programa de Pós-graduação Mestrado em Ensino em Ciências e Saúde, Universidade Federal do Tocantins, Palmas, Brasil.

*Correspondência: Programa de Pós-graduação Mestrado em Ensino em Ciências e Saúde, Universidade Federal do Tocantins, Av. NS 15, 109 Norte, Palmas, Tocantins, Brasil.CEP:77.010-090.e-mail fellipe.cfd@gmail.com

\section{RESUMO}

A sarcopenia está associada a diversas doenças, redução da funcionalidade e prejuízos para qualidade de vida dos gerontes. A associação da sarcopenia com funcionalidade em populações quilombolas tem sido pouco estudada. Este trabalho teve por objetivo verificar a associação entre sarcopenia e funcionalidade em idosas quilombolas e não quilombolas. Estudo transversal realizado com 62 participantes mulheres (variando de 60 a 85 anos), que foram divididas em dois grupos, sendo 39 quilombolas (GQ) e 23 não quilombolas (GC). Para o diagnóstico de sarcopenia foi utilizado o algoritmo proposto pelo European Working Group on Sarcopenia in Older People, a massa muscular foi analisada pelo exame de absortometria de raios-x de dupla energia e a força de preensão palmar por meio do dinamômetro de mão. $\mathrm{O}$ desempenho físico foi analisado pelo teste de velocidade de marcha. Para analisar a capacidade funcional os testes de Katz e Lawton foram utilizados. Foi identificada uma prevalência de sarcopenia de $5,12 \%$ no GQ e de $8,69 \%$ no GC. Há maior proporção de mulheres quilombolas com incapacidade funcional, $100 \%$ delas apresentando dependência para ambas as atividades. A condição de ser quilombola demonstrou maiores prejuízos na capacidade funcional, independentemente de ser acometida por sarcopenia.

Palavras-chave: Sarcopenia; Idosas; Capacidade Funcional.

\section{ABSTRACT}

Sarcopenia is associated with several diseases, reduced functionality and damages to the gerontes' quality of life. The association of sarcopenia with functionality in quilombola populations has been little studied. This study aimed to verify the association between sarcopenia and functionality in elderly quilombolas and non - quilombolas. A crosssectional study was carried out with 62 women participants (ranging from 60 to 85 years old), who were divided into two groups: 39 quilombolas $(G Q)$ and 23 non-quilombolas $(C G)$. For the diagnosis of sarcopenia, the algorithm proposed by the European Working Group on Sarcopenia in Older People was used. Muscle mass was analyzed by examining dual energy x-ray absorptiometry and palmar grip strength using the hand dynamometer. The physical performance was analyzed by gait velocity test. In order to analyze the functional capacity, the Katz and Lawton tests were used. A prevalence of sarcopenia of 5.12\% was found in $G Q$ and $8.69 \%$ in CG. There is a greater proportion of quilombola women with functional disability, $100 \%$ of them presenting dependence for both activities. The condition of being a quilombola showed greater impairments in functional capacity, regardless of whether it was affected by sarcopenia.

Keywords: Sarcopenia; Elderly; Functional capacity.

\section{RESUMEN}

La sarcopenia está asociada a diversas enfermedades, reducción de la funcionalidad y perjuicios para calidad de vida de los gerontes. La asociación de sarcopenia con funcionalidad en poblaciones quilombolas ha sido poco estudiada. Este trabajo tuvo por objetivo verificar la asociación entre sarcopenia y funcionalidad en ancianas 
quilombolas y no quilombolas. Estudio transversal realizado con 62 participantes mujeres (variando de 60 a 85 años), que fueron divididas en dos grupos, siendo 39 quilombolas (GQ) y 23 no quilombolas (GC). Para el diagnóstico de sarcopenia se utilizó el algoritmo propuesto por el European Working Group on Sarcopenia in Older People, la masa muscular fue analizada por el examen de absortometría de rayos $x$ de doble energía y la fuerza de asimiento palmar por medio del dinamómetro de mano. El rendimiento físico fue analizado por la prueba de velocidad de marcha. Para analizar la capacidad funcional se utilizaron las pruebas de Katz y Lawton. Se identificó una prevalencia de sarcopenia del 5,12\% en el GQ y del 8,69\% en el GC. Hay mayor proporción de mujeres quilombolas con incapacidad funcional, $100 \%$ de ellas presentando dependencia para ambas actividades. La condición de ser quilombola demostró mayores pérdidas en la capacidad funcional, independientemente de ser acometida por sarcopenia.

Descriptores: sarcopenia; anciano; Capacidad Funcional.

\section{INTRODUÇÃO}

$\mathrm{Na}$ atualidade, o aumento do número de idosos em escala global se constitui em fato marcante, cuja tendência pode ser observada também no Brasil ao se considerar a população acima dos 60 anos. Segundo o Instituto Brasileiro de Geografia e Estatística, existem 23 milhões de idosos no país, o que corresponde a cerca de dez por cento da população brasileira. Sendo que as mulheres representam $56 \%$ da população total de idosos. Ao analisarmos a raça/etnia desse contingente feminino, aproximadamente de 9,2 \% são da raça negra (IBGE, 2011). Segundo legislação da Fundação Palmares, este grupo étnico-racial é formado segundo critérios de auto-atribuição, com trajetória histórica própria, dotados de relações territoriais específicas, com presunção de ancestralidade negra. Ao analisar a mulher quilombola, estudo demonstrou piores condições de saúde, principalmente relacionados ao status nutricional (SOARES, 2014).

A sarcopenia compreendida como diminuição da massa livre de gordura, força muscular e desempenho físico está relacionada também a outras enfermidades, além da associação com risco de fragilidade, quedas, prejuízos na mobilidade, descontrole glicêmico e metabólico, redução da taxa metabólica basal e capacidade funcional (TYROVOLAS et al.,2015; SILVA-NETO, 2012). Tais condições podem levar a dependência funcional, com maior chance de hospitalização e institucionalização, com ônus financeiro e prejuízos severos à qualidade de vida (KIM, 2015).

Estudos tem relatado a associação de sarcopenia com prejuízos à capacidade funcional dos idosos (VISSER et al. 2010). Nessa direção, em recente pesquisa realizada por Tramontano et al. (2016) foram analisados 222 indivíduos com idade igual ou superior a 65 anos de ambos os sexos, moradores em uma comunidade rural. A sarcopenia foi avaliada utilizando os critérios do International Working Group on sarcopenia, a capacidade funcional foi investigada avaliando dificuldades autorrelatadas dos participantes em realizar uma ou mais atividades básicas ou instrumentais da vida diária. Observaram que, as mulheres sarcopênicas obtiveram piores valores de capacidade funcional.

Apesar de existirem estudos sobre a associação de sarcopenia e funcionalidade em idosos, não há relatos até o momento de nenhuma pesquisa que avalie a relação de sarcopenia e funcionalidade em idosas quilombolas e não quilombolas.

\section{MATERIAIS E MÉTODOS}

A amostra do estudo foi composta por 62 idosas, divididas e dois grupos: um de idosas quilombolas (GQ) composto por 39 moradoras no município de Brejinho de Nazaré, situado a 100 Km de Palmas, Capital do Estado do Tocantins; e o grupo controle (GC) composto de 23 idosas regularmente 
matriculadas na Universidade da Maturidade Polo de Palmas.

Os critérios de inclusão foram obter pontuação igual ou superior a 17 no mini exame de estado mental (MEEM). Excluíram-se do estudo idosas com patologias osteomioarticulares que impedissem a locomoção sem assistência, anormalidade metabólica ou hormonal que sabidamente afeta o sistema muscular e risco cardiovascular que contraindicasse a realização das avaliações propostas no estudo.

As participantes responderam a um questionário para obtenção de informações referentes a dados socioeconômicos como: idade, sexo e escolaridade. Adicionalmente, o nível de atividade física das participantes foi mensurado por meio do IPAQ-versão curta (International Physical Activity Questionnaire) conforme protocolo (MASUDO, 2001). Todas as idosas assinaram o Termo de Consentimento Livre e Esclarecido e este projeto foi aprovado pelo Comitê de Ética em Pesquisa da UFT (protocolo 045/2014).

Quanto às medidas antropométricas, foram mensurados peso, estatura e, posteriormente, calculado o índice de massa corporal (IMC). O diagnóstico da sarcopenia foi feito com base na classificação proposta pelo European Working Group on Sarcopenia in Older People (CRUZ-JENTOFT et al.,2010).

O exame de absortometria de raio-x de dupla energia (DXA) forneceu dados de massa livre de gordura apendicular e percentual de massa gorda. A baixa massa livre de gordura apendicular (MLGA) foi identificada de acordo com ponto de coorte proposto por Baumgartner et al. (1998), o qual definem $\leq 5,5$ $\mathrm{kg} / \mathrm{m} 2$ para mulheres.

A força de preensão palmar (FPP) foi mensurada utilizando o dinamômetro modelo Sahean, sendo classificadas com baixa força muscular valores $\leq 20 \mathrm{Kg} / \mathrm{f}$. No teste de velocidade de marcha (VM) as idosas percorreram uma distância de 3 metros, valores $\leq 0,8 \mathrm{~m} / \mathrm{s}$ foram considerados como prejuízo no desempenho físico (CRUZ-JENTOFT et al.,2010).

Os testes de Katz e Lawton avaliaram a capacidade de realizar atividades básicas e instrumentais da vida diária, respectivamente, classificando as idosas como dependentes ou independentes (KATZ, 1963; LAWTON, 1969).

Dentre as análises estatísticas, foi conduzido para analisar as normalidades das variáveis dependentes pelo teste de Shapiro Wilks. Os dados foram apresentados através da estatística descritiva, utilizando-se os procedimentos de média e desvio padrão. O software SPSS versão 20.0 foi utilizado para realização de todas as análises.

\section{RESULTADOS E DISCUSSÃO}

A idade média do GQ foi de 65,58 \pm 6,67 anos e do GC 64,86 $\pm 6,67$ anos. Em relação a escolaridade, no GQ $31,58 \%$ eram analfabetas, enquanto no GC 100\% tinham pelo menos 1 ano em instituição de ensino. Esses dados corroboram com a literatura que, apontam baixa escolaridade em idosos quilombolas, em especial nas mulheres (FERREIRA, 2014).

Em relação ao nível de atividade física, para ambos os grupos a maior parte das idosas foram identificadas como ativas e muito ativas, no GQ $87,17 \%$ e GC $65,21 \%$. O nível de atividade física é considerado um fator de proteção para 0 desenvolvimento da sarcopenia em idosos (KIM, 2015).

Ambos os grupos foram considerados com sobrepeso de acordo com IMC, o GQ apresentou $27,17 \pm 4,4$ e o GC $27,79 \pm 4,0$. Estudos anteriores verificaram uma associação negativa de sarcopenia e IMC, onde os sarcopênicos apresentavam valores inferiores, no entanto, nesse estudo não foi possível inferir essa afirmação. Isso pode ser explicado parcialmente pelo alto percentual de gordura 
encontrado em ambos os grupos, média de 40,54 46,46 no GQ e de $42,57 \pm 6,28$ no GC (CRUZ-JENTOFT et al.,2010).

A prevalência de sarcopenia encontrada na população estudada foi de 5,12\% (n=2) no GQ e de 8,69\% ( $\mathrm{n}=2$ ) no GC. Outros estudos reportando prevalência de sarcopenia em idosos quilombolas e não quilombolas corroboram com nossos achados (MALMSTRON et al.,2013; VAN KAN et al.,2009). A identificação de idosas com baixa FPP foi encontrada em $41 \%(n=16)$ no GQ e $34,78 \%(n=8)$ no GC, essa medida é um importante marcador de prognóstico para o desenvolvimento da sarcopenia. A força muscular declina mais rapidamente do que a massa muscular, sugerindo que ao envelhecer ocorre um comprometimento da qualidade do músculo. Ademais a literatura ressalta que manter ou ganhar massa muscular não previne o declínio da força muscular. Ainda, a baixa FPP é tida como um fator de prognóstico para quedas, comprometimento cognitivo, hospitalização, doenças cardiovasculares e mortalidade (SILVA-NETO et al., 2016). Portanto, medidas de prevenção e reabilitação para uma FPP adequada são necessárias.

A independência funcional é adjuvante para a manutenção do envelhecimento ativo. As idosas quilombolas (GQ) apresentaram maior dependência do que as idosas não quilombolas (GC). Nas atividades básicas da vida diária (ABVD`S) as idosas quilombolas apresentaram 5,38\% (n=6) e nas atividades instrumentais da vida diária (AIVD's) $33,33 \%(n=13)$ de incapacidade. Todas as idosas do GC apresentaram independência tanto para ABVD`s como AIVD`s. Esses dados corroboram com pesquisa anterior que demonstraram incapacidade funcional em idosos de zona rural, isso pode ser explicado pela baixa escolaridade e menor acesso às ações de promoção, prevenção e cuidados de saúde. Destacamos que isso não é consenso na literatura, maiores investigações devem ser realizadas para verificar a associação dessa dependência com outras variáveis (TAVARES et al.,2011).

A dependência no GQ para realização das ABVD'S foram encontradas nas funções vestir-se e transferência ambas com 15,38\% (n=6). Para as tarefas de AIVD's observamos a incapacidade nas tarefas de usar telefone $23,07 \%(\mathrm{n}=9)$, fazer compras $2,56 \%$ $(\mathrm{n}=1)$, trabalho doméstico $25,64 \%(\mathrm{n}=10)$ e tomar medicações 2,56\% ( $\mathrm{n}=1)$. As idosas sarcopênicas quilombolas $\quad(\mathrm{n}=2)$ apresentaram $100 \%$ de dependência para ambas as atividades. Maiores prevalências de dependência ocorreram nas AIVDs, pois estas atividades exigem maior integridade física e cognitiva comparada às ABVD's (MILAN et al.,2010). Nesse sentido, destacamos à importância da utilização da escala hierárquica de capacidade funcional, que considera a perda gradual das funções com o envelhecimento, em destaque nas populações com limitado acesso aos serviços de saúde, como as idosas quilombolas.

\section{CONCLUSÃO}

Em conclusão, a identificação da prevalência de sarcopenia independente da raça e etnia é adjuvante em idosas. No entanto, maiores prejuízos funcionais foram encontrados nas idosas quilombolas $\mathrm{e}$ sarcopênicas quilombolas. Destacamos que é importante verificar de forma hierárquica a perda de capacidade funcional concomitante com as variáveis de sarcopenia. Essa é uma questão emergencial, pois a incapacidade e sarcopenia estão associados a vários resultados adversos à saúde. Sobretudo, é preciso reconhecer a necessária proteção à saúde de populações minoritárias, visto que os estudos que analisam idosas quilombolas são escassos. Atividades que promovam a manutenção dos altos níveis de atividade física de ambos os grupos devem ser realizadas. Uma estratégia pode ser o exercício 
resistido, como opção para a prevenção e tratamento da sarcopenia, adequados valores de força muscular e capacidade funcional em idosas quilombolas e não quilombolas.

Pesquisas futuras são necessárias para melhor constituir uma definição operacional de sarcopenia na população brasileira, dos quais sugerimos que devem ser integrados na prática clínica para adequado diagnóstico e avaliação do seu impacto na capacidade funcional, em especial nas idosas quilombolas.

\section{AGRADECIMENTO}

O presente trabalho foi realizado com o apoio da UFT, da Universidade da Maturidade e da Associação dos Mini e Pequenos Produtores das Comunidades Quilombolas de Brejinho de Nazaré TO.

Todos os autores declararam não haver qualquer potencial conflito de interesses referente a este artigo.

\section{REFERÊNCIAS}

BAUMGARTNER, R. N.; KOEHLER, K. M.; GALLAGHER, D. Epidemiology of sarcopenia among the elderly in New Mexico. Am J Epidemiol. V.147, p.755-763, 1998

BRASIL. Fundação Palmares. Quilombolas. Disponível em: http://www.palmares.gov.br. [Acesso em 2015 FEV 17]

CRUZ-JENTOFT, A. J.; BAEYENS, J. P.; BAUER, J. M. Sarcopenia: European consensus on definition and diagnosis. Age. Ageing. V.39, p. 412-413, 2010.

FERREIRA, A. E.; CASTILHO, S. D. Reflexões sobre a educação escolar quilombola. RP3-Revista de Pesquisa em Políticas Públicas. V.1, n.1, 2014.

IBGE - Instituto Brasileiro de Geografia e Estatística. Pesquisa Nacional por Amostra de Domicílios (PNAD) Sinopse do censo demográfico: 2010/IBGE. Rio de Janeiro: IBGE, 2011.

KATZ, S.; FORD, A. B.; MOSKOWITZ, R. W. Studies of illness in the aged: the index of ADL: a standardized measure of biological and psychosocial function. JAMA. V.185, p.914-919, 1963.

KIM, T. N.; CHOI, K. M. The Implications of Sarcopenia and Sarcopenic Obesity on Cardiometabolic Disease. J Cell Biochem. V.116, n.7, p.1171-1178, 2015.

LAWTON, M. P.; BRODY, E. M. Assessment of older people: self-maintaining and instrumental activities of daily living. Gerontologist. V.9, p.179186, 1969.

MALMSTROM, T. K.; MILLER, D. K.; HERNING M. M.; MORLEY, J. E. Low appendicular skeletal muscle mass (ASM) with limited mobility and poor health outcomes in middle-aged African Americans. J Cachexia Sarcopenia Muscle. V.4, n.3, p.179-186, 2013.

MATSUDO, S. M.; MATSUDO, V. K. R.; BARROS NETO, T. L. Atividade física e envelhecimento: aspectos epidemiológicos. Rev Bras Med Esporte. V.2, p.2-13, 2001.

MILLÁN-CALENTI, J. C.; TUBÍO, J.; PITAFERNÁNDEZ， S.; GONZÁLEZ-ABRALDES， I.; LORENZO, T.; FERNÁNDEZ- ARRUTY, T.; MASEDA, A. Prevalence of functional disability in activities of daily living (ADL), instrumental activities of daily living (IADL) and associated factors, as predictors of morbidity and mortality. Archives of gerontology and geriatrics. V.5, n.3, p.306-310, 2010 .

SILVA NETO, L. S.; KARNIKOWISKI, M. G.; TAVARES, A. B.; LIMA, R. M. Associação entre sarcopenia, obesidade sarcopênica e força muscular com variáveis relacionadas de qualidade de vida em idosas. Rev Bras Fisioter. V.16, n.5, p.360-367, 2012.

SILVA NETO, L. S. S.; KARNIKOWSKI, M. G.; OSÓRIO, N. B.; PEREIRA, L. C.; MENDES, M. B.; GALATO, D.; MATHEUS, J. P. C. Association between sarcopenia and quality of life in quilombola elderly in Brazil. International journal of general medicine. V.9, p.89, 2016.

SOARES, D. A.; BARRETO, S. M. Sobrepeso e obesidade abdominal em adultos quilombolas, Bahia, Brasil Overweight and abdominal obesity in adults in a quilombo community in Bahia State, Brazil El sobrepeso y la obesidad abdominal en adultos. Cad. Saúde Pública. V.30, n.2, p.341-354, 2014.

TAVARES, D. M. D. S.; JUNIOR, G.; AUGUSTO, S.; DIAS, F. A.; SANTOS, N. M. D. F.; OLIVEIRA, P. B. D. Qualidade de vida e capacidade funcional de 
idosos residentes na zona rural. Rev. RENE. V.12(esp), 895-903, 2011.

TRAMONTANO, A. Prevalence of sarcopenia and associated factors in the healthy older adults of the Peruvian Andes. Archives of Gerontology and Geriatrics. V.68, p.49-54, 2016.

TYROVOLAS, S.; KOYANAGI, A.; OLAYA, B.; AYUSO-MATEOS, J. L.; MIRET, M.; CHATTERJI, $\mathrm{S}$. The role of muscle mass and body fat on disability among older adults: A cross-national analysis. Exp Gerontol. V.3, n.69, p.27-35, 2015.
VAN KAN, G. A.Epidemiology and consequences of sarcopenia. JNHA-The Journal of Nutrition, Health and Aging. V.13, n.8, p.708-712, 2009.

VISSER, M.; GOODPASTER, BH.; KRITCHEVSKY, S. B.; NEWMAN, A. B.; NEVITT, M.; RUBIN, S. M.; SIMONSICK, E. M.; HARRIS, T. B. Muscle mass, Osteoporos Int (2010) 21:543-559 557 muscle strength, and muscle fat infiltration as predictors of incident mobility limitations in wellfunctioning older persons. J Gerontol Ser A Biol Sci Med Sci. V.60, p.324-333, 2005. 